\title{
Thunderclap headache triggered by micturition: responsive to nimodipine
}

\author{
Yuan-Yuan Han $\cdot$ Wei Gui $\cdot$ Jin Zhu • \\ Yi-Min Liu $\cdot$ Kai Wang $\cdot$ Yu Wang
}

Received: 26 May 2011/Accepted: 9 July 2011/Published online: 19 August 2011

(c) The Author(s) 2011. This article is published with open access at Springerlink.com

\begin{abstract}
Primary thunderclap headache (TCH) is a rare condition, of which the onset can be triggered by coughing, exercise, and sexual activity. Micturition is a recognized trigger of secondary $\mathrm{TCH}$ with pheochromocytoma in bladder, but not of primary $\mathrm{TCH}$. We describe a patient with an apparent primary $\mathrm{TCH}$, which repeatedly occurred immediately after micturition until she achieved a therapeutic dosage of nimodipine.
\end{abstract}

Keywords Thunderclap headache $\cdot$ Micturition ·

Nimodipine · Vasospasm

\section{Introduction}

Thunderclap headache (TCH) is a hyper-acute, severe headache that reaches maximum intensity at onset. This type of headache is frequently described by patient as being 'the worst headache of his or her life'. The term $\mathrm{TCH}$ was first used in the description of the headache caused by unruptured intracerebral aneurysms [1]. Because of its hyper-acute and severe characteristics, it is described as thunderclap [2]. TCH could be the conditions secondary to subarachnoid hemorrhage, unruptured

Y.-Y. Han · W. Gui · J. Zhu · Y.-M. Liu · Y. Wang ( $ه)$

Department of Neurology, The First Hospital of Anhui Medical

University, Jixi Road 218, Hefei 230022, China

e-mail: yw4d@hotmail.com

K. Wang $(\bowtie)$

Neuropsychology Group, Anhui Medical University Institute

of Neurology, Jixi Road 218, Hefei 230022, China

e-mail: wangkai1964@sina.com intracranial aneurysm, cerebral venous sinus thrombosis, cervical artery dissection, spontaneous intracranial hypotension, acute hypertensive crisis, or a third ventricle colloid cyst [3]. But primary TCH has been described as a distinct entity when the known causes have been excluded. A primary $\mathrm{TCH}$ may be triggered by coughing, exercise and sexual activity and thus be classified as primary cough headache, primary exertional headache and primary headache associated with sexual activity [4]. There are also micturition-triggered TCHs though these conditions are very rare, but all the reported cases were found attributed to bladder pheochromocytoma and the headache symptom was associated with elevation of blood pressure following micturition $[5,6]$. Here, we report, for the first time, a case of recurrent TCH triggered exclusively by micturition with neither pheochromocytoma in bladder nor elevation of blood pressure following micturition.

\section{Case report}

A 52 year-old woman presented with episodes of suddenbursting headache immediately after micturition for the last 4 days before admission to our department. She described the onset of headache as sudden, severe, and as if she had been 'hit in the head by a car'. She characterized it as a distending headache which first occurred on the bilateral temporal region and progressively spread to all the head within $30 \mathrm{~s}$. The headache reached its peak almost at onset and was rated by her as 9/10 in severity. The headache was accompanied by nausea and vomiting, but not by sense of vertigo or limb incoordination. It lasted for $1-2 \mathrm{~h}$ and disappeared gradually. There was no personal history of headache or other neurological disorders. She had been 
treated for migraine at a community hospital, but oral pain relievers failed to ease the pain.

On examination, she was alert, oriented and anxious, with a blood pressure (BP) of $108 / 64 \mathrm{mmHg}$, a regular heartbeat of 68 beats/minute, and a temperature of $36.8^{\circ} \mathrm{C}$. Her neurological and general examination did not reveal any pathologic findings. For several times during headache attacks immediately after micturition, her BP was monitored to be in normal range.

Findings from laboratory surveys, including antinuclear antibody, C-reactive protein level, erythrocyte sedimentation rate, anticardiolipin antibody, anti-neutrophil cytoplasmic antibodies, urine tests, thyroid functions, lupus anticoagulant, serum catecholamine, were either negative or within normal limits.

Cerebrospinal fluid (CSF) examination, performed $2 \mathrm{~h}$ after one headache attack, had a normal opening pressure, revealed clear fluid with a leukocyte count of $3 / \mathrm{mm}^{3}$, a protein level of $0.39 \mathrm{~g} / \mathrm{l}$ (normal range, $0.15-0.45 \mathrm{~g} / \mathrm{L}$ ), and a glucose level of $3.2 \mathrm{mmol} / \mathrm{L}$ (normal range, 2.5-4.4 mmol/L). CSF viral antibody test revealed negative for all the familiar virus including hepatitis B and C, cytomegalovirus, herpes simplex virus, Epstein-Barr virus.

Brain computerized tomography (CT) scan, magnetic resonance imaging (MRI), and magnetic resonance angiography (MRA) showed no signs of intracerebral hemorrhage, subarachnoid hemorrhage, infarction, thrombosis, dissection or aneurysm of the extracranial or intracranial arteries. The contrast-enhanced CT scan of the abdomen revealed no abnormalities. Electroencephalogram (EEG) was normal. Testing for anxiety and depressive disorders was conducted in the headache-free time among the attacking days, revealing a score of 16 on the Hamilton anxiety scale (HAMA) (mild anxiety $\geq 14$ ) and 9 on the Hamilton depression scale (HAM-D) (mild depression $>7$ ) [7, 8].

Antianxiety treatment with flupentixol and melitracen tablets (one tablet contains $0.5 \mathrm{mg}$ flupentixol and $10 \mathrm{mg}$ melitracen) at a dosage of one tablet in the morning and one tablet at noon, together with antalgesic, pregabalin, at a dosage of $150 \mathrm{mg}$ daily, did not prevent the recurrence of micturitional headaches. During this time, she would micturate two to three times a day and each micturition was followed by a TCH. One week later, antianxietic and antalgesic were substituted with oral nimodipine $60 \mathrm{mg}$ every $6 \mathrm{~h}$ and the intensity of the micturitional headaches gradually became weaker on the first day of nimodipine taking and no further micturitional headache attacks were noted on the second day. The patient was then discharged home in stable condition. Nimodipine treatment was tapered off gradually in the following 6 weeks, and no headache recurred. Six-month follow-up revealed that the patient's condition was very well except occasional slight headaches when she met cold-wind.

\section{Discussion}

TCH is a severe, hyper-acute headache of the suddenbursting type like a 'clap of thunder', of which the pain intensity reaches a peak at onset usually within $60 \mathrm{~s}$. TCH is considered to have two types: primary $\mathrm{TCH}$ and secondary $\mathrm{TCH}$. Secondary TCH could be attributed to vascular causes including subarachnoid hemorrhage (SAH), cerebral venous sinus thrombosis (CVST), pituitary apoplexy, ischemic stroke, severe hypertension crisis, and nonvascular causes including spontaneous intracranial hypotension, third ventricle colloid cyst, intracranial infections [3]. Our MRI, MRA, and CSF examinations had excluded these possible underlying causes in this patient. In very rare situations, micturition can also trigger suddenbursting severe headache in patients, but all these reported cases were accompanied with pheochromocytoma in bladder $[6,9,10]$ and the headaches were usually associated with elevations of blood pressure following micturition [5]. The contrast-enhanced abdomen CT scan and the unelevated blood pressure during headache attack had also excluded the underlying causes of bladder pheochromocytoma. The headache in our patient seems in consistent with the presentations of primary TCH. Apart from the triggering factor micturition, the headache of our patient met the diagnostic criteria for primary $\mathrm{TCH}$ : (i) Severe head pain, (ii) sudden onset, reaching maximum intensity in $<1 \mathrm{~min}$, lasting from $1 \mathrm{~h}$ to 10 days, (iii) Does not recur regularly over subsequent weeks or months, and (iv) Not attributed to another disorder. Though evidence that thunderclap headache exists as a primary condition is poor, primary TCH has been described as a distinct entity [4]. A primary TCH may occur spontaneously while the patient is at rest or during light activities or may only be triggered by exertion, sexual activity, coughing and thus be classified as primary cough headache, primary exertional headache and primary headache associated with sexual activity respectively. The TCH in our patient was exclusively triggered by micturition and there was no serious underlying pathology identified to explain this $\mathrm{TCH}$, thus it might be plausible to consider the micturitional headache as 'primary'. This micturitional headache is an unusual headache not defined by the ICHD-II, therefore the reporting of this case might be helpful for the more detailed headache classification in the future.

Our patient's response to nimodipine was dramatic and impressive during the time when headaches were still worsening. The close chronological concordance between headache relief and initiation of oral nimodipine was in favor of the direct therapeutic effect of nimodipine. Indeed, nimodipine is currently recommended in aneurysmal subarachnoid hemorrhage in order to prevent vasospasm based on its role in the relaxation of the smooth muscle of arterial 
blood vessel walls [11]. Thus, the recurrent micturitional $\mathrm{TCH}$ in our patient might be associated with cerebral vasospasm though the vasospasm was not visible by MRA. Efficacy of nimodipine was reported in primary TCH [12] as well as in primary bathing TCH [13] with vasospasm detected or non-detected. It has been suggested that primary recurrent $\mathrm{TCH}$ can be divided in two groups: one with diffuse vasospasm and the others without any visible vasospasm by MRA, and primary $\mathrm{TCH}$, to some extent, share the same spectrum with reversible cerebral vasoconstriction syndrome (RCVS) based on their similar clinical features and the same rate of ischemic complications $[14,15]$. The therapeutic efficacy of nimodipine in vasospasm associated primary TCH and RCVS as well as in our micturitional headache may suggest a similar pathogenic mechanism of vasospasm for both conditions. Unfortunately, a late control MRA or transcranial Doppler was not conducted to show any presence of late vasospasm eventually in this patient. Recently, it has been shown that, in some patients, the maximal vasospasm detected by transcranial Doppler or by MRA is respectively at 18-25 days and at $16 \pm 10$ days after TCH onset $[16,17]$. The pathophysiological relationship between micturition and cerebral vascular change is poorly understood and speculative. There is anecdotal evidence suggesting that abdominal support, voluntary diaphragm and abdominal muscle co-activation, is necessary for effective voiding, this results in an alteration of intra-abdominal pressure. This was suggested to have causative relationship to TCH triggered by singing [18]. This intra-abdominal pressure alteration may trigger an unclear neural reflex resulting in a cerebral vascular change.

Involvement of non-organic, psychiatric factors should also be considered to contribute to the recurrent headaches, as a mild depressive (HAM-D $=16$ ) and a mild anxiety disorder $(\mathrm{HAM}-\mathrm{A}=9)$ were revealed and a distinct phobia for micturition also found in our patient. Whereas, the negative result of the antipsychotic treatment seemly indicated that the psychiatric factors may not play a critical role in the headache recurrence. Further case reports may help to elucidate the possible role of psychiatric factors in the micturitional headache.

In summary, we treated a patient with recurrent TCH apparently triggered by micturition without any underlying organic causes, which was responsive to nimodipine dramatically. We hypothesize that the pathophysiology of this primary micturitional headache is associated with cerebral vasospasm induced by neural reflex after the intraabdominal pressure is altered.

Ethical standards: The human studies have been approved by the Anhui Medical University ethics committee and have therefore been performed in accordance with the ethical standards laid down in the 1964 Declaration of
Helsinki. Informed consent was obtained from the patient in this report prior to the related test and report.

\section{Conflict of interest None.}

Open Access This article is distributed under the terms of the Creative Commons Attribution License which permits any use, distribution and reproduction in any medium, provided the original author(s) and source are credited.

\section{References}

1. Day JW, Raskin NH (1986) Thunderclap headache: symptom of unruptured cerebral aneurysm. Lancet 2(8518):1247-1248

2. Schwedt TJ (2007) Clinical spectrum of thunderclap headache. Expert Rev Neurother 7(9):1135-1144

3. Schwedt TJ, Matharu MS, Dodick DW (2006) Thunderclap headache. Lancet Neurol 5(7):621-631

4. Headache Classification Subcommittee of the International Headache Society (2004) The international classification of headache disorders: 2nd edition. Cephalalgia 24(Suppl 1):S9S160

5. Fujishima S, Abe I, Kaseda S, Koga T, Hirano H, Hamada T, Oniki H, Onoyama K, Fujishima M (1997) Ambulatory blood pressure monitoring in diagnosing a pheochromocytoma of the urinary bladder. A case report. Angiology 48(7):655-658

6. Im SH, Kim NH (2008) Thunderclap headache after micturition in bladder pheochromocytoma. Headache 48(6):965-967

7. Hamilton M (1959) The assessment of anxiety states by rating. $\mathrm{Br}$ J Med Psychol 32(1):50-55

8. Hamilton M (1960) A rating scale for depression. J Neurol Neurosurg Psychiatry 23:56-62

9. Heo YE, Kwon HM, Nam HW (2009) Thunderclap headache as an initial manifestation of phaeochromocytoma. Cephalalgia 29(3):388-390

10. Watanabe M, Takahashi A, Shimano H, Hara H, Sugita S, Nakamagoe K, Tamaoka A (2010) Thunderclap headache without hypertension in a patient with pheochromocytoma. J Headache Pain 11(5):441-444

11. Zuber M, Touze E, Domigo V, Trystram D, Lamy C, Mas JL (2006) Reversible cerebral angiopathy: efficacy of nimodipine. J Neurol 253(12):1585-1588

12. Lu SR, Liao YC, Fuh JL, Lirng JF, Wang SJ (2004) Nimodipine for treatment of primary thunderclap headache. Neurology 62(8):1414-1416

13. Liao YC, Fuh JL, Lirng JF, Lu SR, Wu ZA, Wang SJ (2003) Bathing headache: a variant of idiopathic thunderclap headache. Cephalalgia 23(9):854-859

14. Chen SP, Fuh JL, Lirng JF, Chang FC, Wang SJ (2006) Recurrent primary thunderclap headache and benign CNS angiopathy: spectra of the same disorder? Neurology 67(12):2164-2169

15. Ferrante E, Tassorelli C, Rossi P, Lisotto C, Nappi G (2011) Focus on the management of thunderclap headache: from nosography to treatment. J Headache Pain 12(2):251-258

16. Chen SP, Fuh JL, Chang FC, Lirng JF, Shia BC, Wang SJ (2008) Transcranial color doppler study for reversible cerebral vasoconstriction syndromes. Ann Neurol 63(6):751-757

17. Chen SP, Fuh JL, Wang SJ, Chang FC, Lirng JF, Fang YC, Shia BC, Wu JC (2010) Magnetic resonance angiography in reversible cerebral vasoconstriction syndromes. Ann Neurol 67(5):648-656

18. Kim YI, Lee SJ, Lee KS, Park JW, Kim JS (2008) Recurrent thunderclap headache triggered by singing. Eur J Neurol 15(12):e116-e117 